almost identical presentation. ${ }^{1}$ The baby was born to a 41 year old Pakistani woman, gravida 12 para 6 . The parents were first cousins. The unfortunate obstetric history included four first trimester spontaneous abortions and a 29 week stillbirth. Another baby, an anencephalic, died in the neonata period. Finally a girl, born at full term, died at 4 weeks of age after a three day illness characterised by fever, convulsions, and hepatosplenomegaly. Postmortem examination showed excessive deposition of sphingomyelin in the spleen.

In a subsequent pregnancy hydramnios was noted at 26 weeks' gestation. An ultrasound scan showed fetal ascites but no structural abnormalities. The baby was born at 34 weeks' gestation weighing $2590 \mathrm{~g}$. There was gross ascites and hepatosplenomegaly. Jaundice was evident on the first day of life (tota bilirubin concentration $111 \mu \mathrm{mol} / 1$, conjugated bilirubin $83 \mu \mathrm{mol} / \mathrm{l})$. During the nex two weeks there was clinical and laboratory evidence of continuing hepatic dysfunction and the baby died from liver failure at 19 days.

Investigations excluded other causes of feta ascites and neonatal liver disease. Peripheral leucocyte enzyme assays were normal. Bone marrow aspirate showed foamy histiocytes. A percutaneous postmortem splenic biopsy specimen showed accumulation of sphing omyelin. Partial deficiency of sphingomyelinase was found in cultured skin fibroblasts The fibroblasts showed appreciably reduced intracellular esterificaton of exogenous lipoprotein derived cholesterol, consistent with Niemann-Pick disease type C.

Clearly this disease should be considered in the differential diagnosis of fetal ascites and conjugated hyperbilirubinaemia in the neonate. Whether, in our case, any of the previous abortions or the stillbirth was affected is speculative. Undoubtedly, increasing experience with ultrasound will assist in antenatal recognition of the disease. With regard to postnatal investigation our case emphasise the importance of skin fibroblast culture. In addition to studying cholesterol esterification partial deficiency of fibroblast sphingomyelinase may be found, whereas peripheral leucocyte levels are typically normal.

D J MANNING W I J PRICE Neonatal Intensive Care Unit, fessop Hospital for Women, Leavygreave Road,

1 Maconochie IK, Chong S, Mieli-Vergani G Lake BD, Mowat AP. Fetal ascites: an unusua presentation of Niemann-Pick disease type C. Arch Dis Child 1989;64:1391-3.

The school health service through parents' eyes

SIR,-We read with interest the personal paper on the school health service by Dr Perkins and would like to make some comments. ${ }^{1}$

It is important to have a clearly defined function communicated to all users of the service. At the moment many teachers misunderstand and have inappropriate expectations of what we should achieve, which leads to disappointment and downgrading of the service in teachers' eyes that is communicated to parents.
Inadequate facilities are provided in many schools, which must communicate lack of interest in the service to parents and children.

Difficulties arise because of rules that have developed for historical reasons_-for example, we have little access to simple investigation and also most things must be referred back or through general practitioners before action can be taken. This apparent lack of authority even in areas of special interest must convey low status.

Certainly communication could be improved by having a named doctor and nurse visiting the school regularly throughout the school year. In order to do the job efficiently we must have a clearly defined role about which all users have appropriate expectations. We should be able to fulfil that role in as direct a way as possible to be seen as having appropriate authority. Above all it must be seen as a useful service to parents and teachers and children. These are the personal views of the signatories.

P BLACKWELL,
S SIKKA,
G N DAVIES,
C RAVINDRANATH
Community Services (Child Health),
Grimsby District General Hospital,
Grimsby, South Humberside
DN33 $2 B A$

1 Perkins ER. The school health service through parents' eyes. Arch Dis Child 1989;64:1088-91.

\section{Chlamydia trachomatis in infants: a prospective study}

SIR,-We were interested to read the paper by Preece $e t$ al dealing with chlamydial infection in children but feel that some important points may have been omitted. ${ }^{1}$ In infants, Chlamydia trachomatis infects multiple sites including the rectum and vulva. ${ }^{2}$ Screening from conjunctiva and pharnyx alone may underestimate transmission from infected mothers. Where a more complete screening practice has been used, longer carrier status has been shown. ${ }^{3}$ Failure to recognise the reality of genital carriage, and especially the declaration that a child is free from infection on the basis of limited sampling, carries serious social and legal implications, as some practitioners regard the presence of vaginal chlamydia as an indicator of sexual abuse. ${ }^{4}$ Topical treatment of chlamydial conjunctivitis does not clear the child of the pathogen at other sites and leaves it vulnerable to completely avoidable respiratory infection.

Finally, we wish to challenge the assumption that antenatal screening for chlamydia is not cost effective. No account has been taken of an important part of the equation, namely the pregnant woman herself and of the morbidity she may avoid by receiving treatment earlier rather than later.

K POWELL G W MIDDLETON G E FORSTER The Whitechapel Clinic, The London Hospital, Whitechapel, London EI IBB

1 Preece PM, Anderson JM, Thompson RG. Chlamydia trachomatis in infants: a prospective study. Arch Dis Child 1989;64:525-9.

2 Schacter J, Grossman M, Holt J, et al. Infection with Chlamydia trachomatis: involvement of multiple anatomic sites in neonates. $\mathcal{F}$ Infect Dis 1979;139:232-4.
3 Bell TA, Stamm WE, Kuo C-C, et al. Chronic Chlamydia trachomatis infections in infants. In: Oriel D, Ridgway G, Schacter J. Chlamydial infections. Cambridge: Cambridge University Press, 1986:305-8.

4 Ingram DL, White ST, Occhiuti AR, et al. Childhood vaginal infections: association of chlamydia trachomatis with sexual contact. Pediatr Infect Dis 1986;5:226-9.

Drs Preece, Anderson, and Thompson comment: Our recent paper explored the postnatal morbidity in infants associated with perinatal transmission of maternal $C$ trachomatis infection. While it is interesting to document the persistence of rectal and vaginal carriage this was not our primary aim, and we agree that studies to document such carriage and persistence of infection are required to distinguish from later acquisition.

We agree that in clinical practice, oral erythromycin is now the treatment of choice in all chlamydial infections in children.

Finally, our statement about cost effectiveness of screening for maternal infection was based on the subsequent perinatal and postnatal morbidity in infants. The practicality and effectiveness of such a screening programme is discussed in more detail in our companion paper. ${ }^{2}$ We feel that our data do not support such a programme to protect infants from chlamydial infection for the reasons outlined.

With reference to maternal infection, pregnancy may provide an opportunity to screen for $C$ trachomatis infection in adult women. However, there may be other strategies that will be more logical and cost effective in the management of adult infection. As Drs Powell et al point out we do not address this issue, which needs more extensive and specifically designed studies of the epidemiology of $C$ trachomatis infection in adult women.

1 Rettig PJ. Chlamydial infections in paediatrics: diagnostic and therapeutic considerations. Pediatr Infect Dis 1986;5:158-62.

2 Preece PM, Ades A, Thompson RG, Brooks JH. Chlamydia trachomatis infection in late pregnancy: a prospective study. Paediatric and Perinatal Epidemiology 1989;3:268-77.

Egg and breast milk based nitrogen sources compared

SIR,-In their paper comparing the effects of intravenous Vamin 9 glucose and Vaminolact on the plasma amino acid profile, Puntis et al discuss the possible neurotoxic effects of raised phenylalanine concentrations. ${ }^{1}$ They conclude that Vaminolact is preferable to Vamin 9 glucose because it was associated with less marked increases in plasma phenylalanine concentrations. In support of this view they refer to findings in early treated children with phenylketonuria showing that intellectual outcome is inversely associated with average phenylalanine control during treatment (as well as age at the start of treatment). ${ }^{2}$ I doubt whether it is appropriate to liken the effects of short term changes in phenylalanine in children receiving Vamin 9 glucose (which also caused rises in other amino acids including tyrosine) to the effects of longterm phenylalanine control in phenylketonuria, which is associated with decreased rather than increased concentrations of tyrosine.

It may be more useful to consider the likely effects of the raised phenylalanine concentrations on the uptake of amino acids into the brain. ${ }^{3}$ This will depend upon the plasma concentrations of the other large neutral amino 